\title{
The Design and Evaluation of the Comprehensive Hospitalist Assessment and Mentorship with Portfolios (CHAMP) Ultrasound Program
}

\author{
Benji K. Mathews, MD FACP SFHM ${ }^{1,2 *}$, Kreegan Reierson, MD¹, Khuong Vuong, MD¹, Ankit Mehta, MBBS, FHM, FACP1 \\ Paula Miller, $\mathrm{MPH}^{3}$, Seth Koenig, MD, FCCP ${ }^{4,5}$, Mangala Narasimhan, DO, FCCP5,6
}

\begin{abstract}
'Department of Hospital Medicine, HealthPartners, Bloomington, Minnesota; ${ }^{2}$ University of Minnesota Medical School, Minneapolis, Minnesota; ${ }^{3}$ Critical Care Research Center, Regions Hospital, Saint Paul, Minnesota; ${ }^{4}$ Long Island Jewish Medical Center of Northwell Health, New Hyde Park, New York; ${ }^{5}$ Department of Medicine, Donald and Barbara Zucker School of Medicine at Hofstra/Northwell, Hofstra University, Hempstead, New York; ${ }^{6}$ Critical Care Medicine, Northwell Health System, New Hyde Park, New York.
\end{abstract}

BACKGROUND: Literature supports the use of point-ofcare ultrasound performed by the treating hospitalist in the diagnosis of common diseases. There is no consensus on the training paradigm or the evaluation of skill retention for hospitalists.

OBJECTIVE: To evaluate the effectiveness of a comprehensive bedside ultrasound training program with postcourse competency assessments for hospitalists.

DESIGN: A retrospective report of a training program with 53 hospitalists. The program consisted of online modules, a 3-day in-person course, portfolios, 1-day refresher training, monthly scanning, and assessments. Hospitalists were rated by using similar pre- and postcourse competency assessments and self-rating parameters during the 3-day and refresher courses.

SETTING: A large tertiary-care center.

RESULTS: Skills increased after the 3-day course from a median preassessment score of $15 \%$ correct (interquartile range [IQR] 10\%-25\%) to a median postassessment score of $90 \%$ (IQR $80 \%-95 \% ; P<.0001)$. At the time of the refresher course, the median precourse skills score had decreased to $65 \%$ correct (IQR 35\%-90\%), which improved to $100 \%$ postcourse (IQR $85 \%-100 \%$; $P<.0001$ ). Skills scores decreased significantly less between the post 3-day course assessment and pre 1-day refresher course for hospitalists who completed portfolios (mean decrease $13.6 \%$ correct; $P<.0001$ ) and/or monthly scanning sessions (mean decrease $7.3 \%$ correct; $P<.0001$ ) compared with hospitalists who did not complete these items.

CONCLUSIONS: A comprehensive longitudinal ultrasound training program including competency assessments improved ultrasound acquisition skills with hospitalists. Skill retention remained high in those who completed portfolios and/or monthly scanning sessions along with a 1-day in-person refresher course. Journal of Hospital Medicine 2018;13:544-550. Published online first February 27, 2018. (c) 2018 Society of Hospital Medicine
$\mathrm{P}$ oint-of-care ultrasound (POCUS) is a valuable tool to assist in the diagnosis and treatment of many common diseases. ${ }^{1-11}$ Its use has increased in clinical settings over the years, primarily because of more portable, economical, high-quality devices and training availability. ${ }^{12} \mathrm{PO}-$ CUS improves procedural success and guides the diagnostic management of hospitalized patients. ,,-12 Literature details the training of medical students, ${ }^{13,14}$ residents, ${ }^{15-21}$ and providers in emergency medicine ${ }^{22}$ and critical care, ${ }^{23,24}$ as well as focused cardiac training with hospitalists. ${ }^{25-27}$ However, no literature exists describing a comprehensive longitudinal training program for hospitalists or skills retention.

*Address for correspondence: Benji K. Mathews, MD, FACP, SFHM, CLHM Regions Hospital, 640 Jackson Street, Mail Stop 11109E, Saint Paul, MN 55101; Telephone: 651-254-9555; Fax: 651-254-9673; E-mail: benji.k.mathews@healthpartners.com

Additional Supporting Information may be found in the online version of this article.

Received: April 3, 2017; Revised: November 15, 2017;

Accepted: November 23, 2017

(C) 2018 Society of Hospital Medicine DOI 10.12788/jhm.2938
This document details the hospital medicine department's ultrasound training program from Regions Hospital, part of HealthPartners in Saint Paul, Minnesota, a large tertiary care medical center. We describe the development and effectiveness of the Comprehensive Hospitalist Assessment and Mentorship with Portfolios (CHAMP) Ultrasound Program. This approach is intended to support the development of POCUS training programs at other organizations.

The aim of the program was to build a comprehensive bedside ultrasound training paradigm for hospitalists. The primary objective of the study was to assess the program's effect on skills over time. Secondary objectives were confidence ratings in the use of ultrasound and with various patient care realms (volume management, quality of physical exam, and ability to narrow the differential diagnosis). We hypothesized there would be higher retention of ultrasound skills in those who completed portfolios and/or monthly scanning sessions as well as increased confidence through all secondary outcome measures (see below).

\section{MATERIALS AND METHODS}

This was a retrospective descriptive report of hospitalists who entered the CHAMP Ultrasound Program. Study participants 
were providers from the 454-bed Regions Hospital in Saint Paul, Minnesota. The study was deemed exempt by the HealthPartners Institutional Review Board. Three discrete 3-day courses and two 1-day in-person courses held at the Regions Hospital Simulation Center (Saint Paul, Minnesota) were studied.

\section{Program Description}

In 2014, a working group was developed in the hospital medicine department to support the hospital-wide POCUS committee with a charter to provide standardized training for providers to complete credentialing. ${ }^{28}$ The goal of the hospital medicine ultrasound program was to establish the use of ultrasound by credentialed hospitalists into well-defined applications integrated into the practice of hospital medicine. Two providers were selected to lead the efforts and completed additional training through the American College of Chest Physicians (CHEST) Certificate of Completion Program. ${ }^{29}$ An overall director was designated with the responsibilities delineated in supplementary Appendix 1. This director provided leadership on group practice, protocols, and equipment, creating the organizational framework for success with the training program. The hospital medicine training program had a 3-day in-person component built off the CHEST Critical Care Ultrasonography Program. ${ }^{24}$ The curriculum was adapted from the American College of Chest Physicians/Société de Réanimation de Langue Française Statement on Competence in Critical Care Ultrasonography. ${ }^{30}$ See Table 1 for the components of the training program.

All components of the training program are required to receive the certificate of completion with the exception of the refresher training. Learner feedback after each 3-day course and refresher training was incorporated into subsequent iterations of the training program. During initial phases, additional hands-on faculty were recruited from emergency medicine and critical care who had extensive experience with bedside ultrasound. Subsequently, faculty consisted of former course participants. All faculty followed a standard set of ultrasound and educational principles to guide the hands-on training of participants (supplementary Appendix 2).

\section{Online Modules}

As a prerequisite to the 3-day introductory course, hospitalists were required to complete modules for precourse knowledge involving a set of focused-topic online reading and videos with quizzes (supplementary Appendix 3).

\section{3-Day In-Person Course with Assessments}

The 3-day course provided 6 hours of didactics, 8 hours of image interpretation, and 9 hours of hands-on instruction (supplementary Appendix 4). Hospitalists first attended a large group didactic, followed by divided groups in image interpretation and hands-on scanning. ${ }^{24}$

Didactics were provided in a room with a 2-screen set up. Providers used 1 screen to present primary content and the other for simultaneously scanning a human model.

Image interpretation sessions were interactive smaller group learning forums in which participants reviewed high-yield im-
TABLE 1. The Components of the Overall Training Program

\begin{tabular}{ll}
\hline Component \\
\hline 1. Online modules \\
\hline 2. 3-day in-person course with pre and post written and skills assessment \\
\hline 3. $\quad$ Portfolio development \\
\hline 4. 1-day refresher training with pre and post skills assessment (optional) \\
\hline 5. Monthly scanning sessions (optional) \\
\hline 6. & Final knowledge and skills assessments \\
\hline
\end{tabular}

ages related to the care of hospital medicine patients and received feedback. Approximately 45 videos with normal and abnormal findings were reviewed during each session.

The hands-on scanning component was accomplished with human models and a faculty-to-participant ratio between 1:2 and 1:3. Human models for this course were paid community models. A variety of ultrasound machine platforms were provided for participants. Learning objectives were clearly delineated prior to each scanning session to ensure the coverage of required content.

\section{Portfolios}

Portfolio development was a key aspect in overall POCUS competency for each participant. The hospital medicine department's required portfolio files are presented in the Figure, with standards coinciding with the quality assurance grading rubric as developed by the POCUS committee at Regions Hospital and described by Mathews and Zwank. ${ }^{28}$ Images taken with real patients were submitted without patient identifiers to a shared online portal. Faculty provided regular cycling feedback by entering the status of submission (accepted or declined) and specific comments on images and interpretations. Learners worked off of the feedback, practiced their skills, and resubmitted files. An image was considered acceptable if it met criteria of depth, axis, and gain and showed the required organ. Participants could use the same patient for different views but could not use the same patient for multiple images of the same view.

Refresher Training: 1-Day In-Person Course with Assessments and Monthly Scanning Sessions (Optional) Only hospitalists who completed the 3-day course were eligible to take the 1-day in-person refresher course (supplementary Appendix 5). The first half of the course incorporated scanning with live human models, while the second half of the course had scanning with hospitalized patients focusing on pathology (pleural effusion, hydronephrosis, reduced left ventricular function, etc.). The course was offered at 3, 6, and 12 months after the initial 3-day course.

Monthly scanning sessions occurred for 2 hours every third Friday and were also available prior to the 1-day refresher. The first 90 minutes had a hands-on scanning component with hos- 
pitalized patients with faculty supervision (1:2 ratio). The last 30 minutes had an image interpretation component.

\section{Assessments}

Knowledge and skills assessment were adapted from the CHEST model (supplementary Appendix 6). ${ }^{24}$ Before and after the 3-day and 1-day in-person courses, the same hands-on skills assessment with a checklist was provided (supplementary Appendix 7). Before and after the 3-day course, a written knowledge assessment with case-based image interpretation was provided (supplementary Appendix 6). A final knowledge and skills assessment was given at either of the in-person courses to those who completed the required components of the training. Passing scores for the final knowledge assessment were established at $85 \%$ items correct by an expert panel by using the Angoff method. ${ }^{31}$ This same standard was applied to the final skills examination. Participants who do not pass the final assessments are provided opportunities for further training and allowed to reattempt the assessments. In this regard, there is a standard training outcome but variances in length of training time for each participant. Pre- and postcourse skills assessments used the same faculty, checklist, and ultrasound device. Raters received an orientation the day prior to each in-person course, reviewing common learner pitfalls, reviewing the checklist, and discussing specific examples.

\section{Measurement}

Participant demographic and clinical information was collected at the initial 3-day course for all participants, including age, gender, specialty, years of experience, and number and type of ultrasound procedures personally conducted or supervised in the past year. For skills assessment, a 20-item dichotomous checklist was developed and scored as done correctly or not done/done incorrectly. This same assessment was provided both before and after each of the 3-day and 1-day courses. A 20-question image-based knowledge assessment was also developed and administered both before and after the 3-day course only. The same 20-item checklist was used for the final skills examination. However, a new more detailed 50-question examination was written for the final examination after the portfolio of images was complete. Self-reported measures were confidence in the use of ultrasound, volume management, quality of physical exam, and ability to narrow the differential diagnosis. Confidence in ultrasound use, confidence in volume management, and quality of physical exam were assessed by using a questionnaire both before and after the 3 -day course and 1-day course. Participants rated confidence and quality on a 5 -point scale, 1 being least confident and 5 being most confident.

\section{Statistical Analysis}

Demographics of the included hospitalist population and pre and post 3-day assessments, including knowledge score, skills score, confidence in ultrasound use, confidence in volume management, and quality of physical exam, were summarized. Values for all assessment variables are presented as percent-

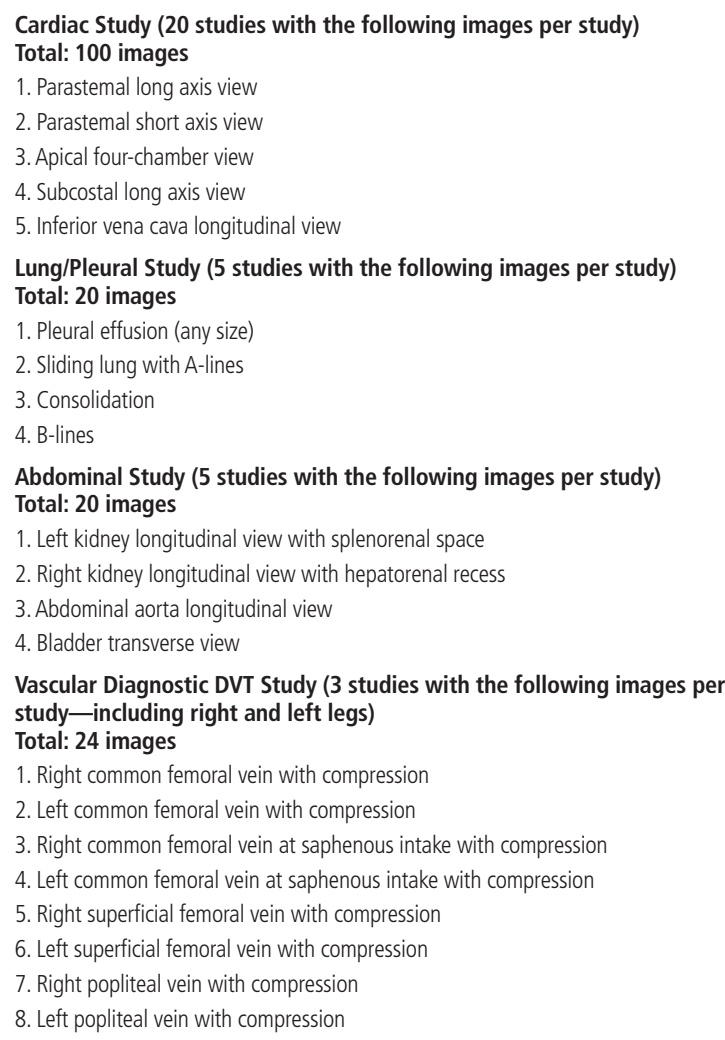

FIG. CHAMP Ultrasound Program Portfolio Requirements

ages. Confidence scores were reported as a percentage of the Likert scale (eg, 4/5 was reported as $80 \%$ ). Skills and written examinations were expressed as percentages of items correct. Data were reported as median and interquartile range or means and standard deviation based on variable distributions. Differences between pre- and postvalues for 3-day course variables were assessed by using 2-sample paired Wilcoxon signed rank tests with a $95 \%$ confidence level.

For the subset of hospitalists who also completed the 1-day course, pre and post 1-day course assessments, including skills score, confidence in ultrasound use, confidence in volume management, and quality of physical exam, were summarized. Differences between pre- and postvalues for 1-day assessment variables were assessed by using 2-sample paired Wilcoxon signed rank tests with a $95 \%$ confidence level.

For hospitalists who completed both the 3-day and 1-day courses, the change in course assessments, including skills score, confidence in ultrasound use, confidence in volume management, and quality of physical exam, was assessed by summarizing the change from post 3-day metrics to pre 1-day metrics (Table 2). The differences between these 2 assessments were evaluated by using 2-sample paired Wilcoxon signed rank tests with a 95\% confidence level. Changes in skills score from post 3-day assessment to pre 1-day assessment were also compared for hospitalists completing any of the portfolio and those completing none, and for hospitalists attending any monthly scanning sessions and those who did not attend any, 
TABLE 2. Difference in Assessment Scores Before and After the 3-Day and 1-Day Courses and Difference in Mean Skills Score Between the Post 3-day and Pre 1-day Assessment by Skills Retention Action

\begin{tabular}{|c|c|c|c|}
\hline Assessment & Median Score Pre (IQR) & Median Score Post (IQR) & $P$ value \\
\hline \multicolumn{4}{|l|}{ Pre to post 3-day course change } \\
\hline Skills (\%) & $15.0(15.0)$ & $90.0(15.0)$ & $<.0001$ \\
\hline Knowledge (\%) & $40.0(20.0)$ & $90.0(15.0)$ & $<.0001$ \\
\hline Confidence in US use (\%) & $20.0(0.0)$ & $60.0(40.0)$ & $<.0001$ \\
\hline Confidence in volume management (\%) & $60.0(40.0)$ & $80.0(0.0)$ & $<.0001$ \\
\hline Quality of physical exam (\%) & $60.0(20.0)$ & $80.0(0.0)$ & $<.0001$ \\
\hline \multicolumn{4}{|l|}{ Pre to post 1 -day course change } \\
\hline Skills (\%) & $65.0(55.0)$ & $100.0(15.0)$ & $<.0001$ \\
\hline Confidence in US use (\%) & $40.0(20.0)$ & $80.0(20.0)$ & $<.0001$ \\
\hline Confidence in volume management (\%) & $40.0(20.0)$ & $80.0(20.0)$ & $<.0001$ \\
\hline \multirow[t]{2}{*}{ Quality of physical exam (\%) } & $40.0(20.0)$ & $80.0(20.0)$ & $<.0001$ \\
\hline & Median Post 3-day (IQR) & Median Pre 1-day (IQR) & $P$ value \\
\hline \multicolumn{4}{|l|}{ Post 3-day to pre 1-day course change } \\
\hline Skills (\%) & $90.0(15.0)$ & $65.0(55.0)$ & $<.0001$ \\
\hline Confidence in US use (\%) & $60.0(40.0)$ & $40.0(20.0)$ & .0058 \\
\hline Confidence in volume management (\%) & $80.0(20.0)$ & $40.0(20.0)$ & $<.0001$ \\
\hline \multirow[t]{2}{*}{ Quality of physical exam (\%) } & $80.0(20.0)$ & $40.0(20.0)$ & $<.0001$ \\
\hline & Mean Skills Post 3-day (SD) & Mean Skills Pre 1-day (SD) & $P$ value \\
\hline \multicolumn{4}{|l|}{ Skills retention action } \\
\hline \multicolumn{4}{|l|}{ Portfolio completed } \\
\hline Any & $92.0(6.5)$ & $78.4(14.0)$ & \\
\hline None & $82.5(6.0)$ & $32.5(10.4)$ & $<.0001$ \\
\hline \multicolumn{4}{|l|}{ Monthly scanning sessions } \\
\hline Any & $94.5(6.3)$ & $87.2(7.9)$ & \\
\hline None & $85.0(6.2)$ & $46.5(19.2)$ & $<.0001$ \\
\hline
\end{tabular}

NOTE: All values are displayed as percentages. Abbreviations: IQR, interquartile range; SD, standard deviation; US, ultrasound.

by using analysis of variance and Scheffe tests.

Multiple linear regression was performed with the change in skills assessment score from postcompletion of the 3-day course to precompletion of the 1-day course as the dependent variable. Hospitalists were split into two age groups (3039 and 40-49) for the purpose of this analysis. The percent of monthly scanning sessions attended, age category, timing of 1-day course, and percent portfolio were assessed as possible predictors of the skills score by using simple linear regression with a $P=.05$ cutoff. A final model was chosen based on predictors significant in simple linear regression and included the percent of the portfolio completed and attendance of monthly scanning sessions.

\section{RESULTS}

\section{Demographics}

Of the 56 3-day course participants, 53 had complete data (Table 3). Three participants with incomplete data completed most of the course but left prior to postcourse assessments and were excluded from the analysis. Twenty-three hospitalists also completed the 1-day in-person course. Seven hospitalists completed the 1-day course 3 months after the initial course, 8 completed it at 6 months, and 8 completed it at 12 months.
Completed portfolios required 164 approved video images. Fifteen of the 23 hospitalists at the 1-day course have started and are working towards completion of the online portfolio, while 9 of the 23 participated in the monthly scanning sessions.

\section{3-Day In-Person Course}

For the 53 hospitalists who completed skills-based assessments, performance increased significantly after the 3-day course. Knowledge scores also increased significantly from preassessment to postassessment. Self-reported confidence ratings for ultrasound use, confidence in volume management, and quality of physical exam all increased significantly from preassessment to postassessment (Table 2).

\section{Refresher Training: 1-Day In-Person Course}

Because the refresher training was encouraged but not required, only 25 of 53 hospitalists, 23 with complete data, completed the 1-day course. For the 23 hospitalists who completed skills-based assessments before and after the 1-day course, mean skills scores increased significantly (Table 2). Self-reported confidence ratings for ultrasound use, confidence in volume management, and quality of physical exam all increased significantly from preassessment to postassessment (Table 2). 
Monthly Scanning Sessions and Portfolio Development

The skills retention from initial course to refresher course by portfolio completion and monthly scanning sessions is shown in Table 2. Multiple regression analysis showed that for every $10 \%$ increase in the percent of monthly sessions attended, the mean change in skills score was $3.7 \%(P=.017)$, and for every $10 \%$ increase in the percent of portfolio completed, the mean change in skills score was $2.5 \%(P=.04)$, showing that both monthly scanning session attendance and portfolio completion are significantly predictive of skills retention over time.

\section{Final Assessments}

Four providers met mastery at initial attempt. No providers to date have needed remediation. Many others are going through different stages of the process and are expected to attain mastery in a short period of time.

\section{DISCUSSION}

This is the first description of a successful longitudinal training program with assessments in POCUS for hospital medicine providers that shows an increase in skill retention with the use of a follow-up course and bedside scanning.

The CHAMP Ultrasound Program was developed to provide hospital medicine clinicians with a specialty focused in-house training pathway in POCUS and to assist in sustained skills acquisition by providing opportunities for regular feedback and practice. Practice with regular expert feedback is a critical aspect to develop and maintain skills in POCUS. ${ }^{32,33}$ Arntfield $^{34}$ described the utility of remote supervision with feedback for ultrasound training in critical care, which demonstrated varying learning curves in the submission of portfolio images. ${ }^{35,36}$ The CHAMP Ultrasound training program provided expert oversight, longitudinal supervision, and feedback for course participants. The educational method of mastery learning was employed by setting minimum standards and allowing learners to practice until they met that standard. ${ }^{37-39}$

This unique program is made possible by the availability of expert-level faculty. Assessment scores improved with an initial 3-day course; however, they also decayed over time, most prominently with hospitalists that did not continue with POCUS scanning after their initial course. Ironically, those who performed more ultrasounds in the year prior to beginning the 3-day course had lower confidence ratings, likely explained by their awareness of their limitations and opportunities for improvement. The incorporation of refresher training to supplement the core 3-day course and portfolio development are key additions that differentiate this training program. These additions and the demonstration of successful training make this a durable pathway for other hospitalist programs. There are many workshops and short courses for medical students, residents, and practicing providers in POCUS. ${ }^{40-43}$ However, without an opportunity for longitudinal supervision and feedback, there is a noted decrease in the skills for participants. The refresher training with its 2 components (1-day in-person course and monthly scanning sessions) provides evidence of the value of mentored training.
TABLE 3. Demographic Characteristics of Hospitalists Completing the 3-Day Training Course

\begin{tabular}{lc}
\hline Characteristic & $\mathbf{N}(\%)$ \\
\hline Age (years) & \\
$20-29$ & $3(5.9)$ \\
$30-39$ & $24(47.1)$ \\
$40-49$ & $15(29.4)$ \\
$50-59$ & $6(11.8)$ \\
$60+$ & $3(5.88)$ \\
\hline Gender & \\
Female & $21(41.2)$ \\
Male & $30(58.8)$ \\
\hline Years of practice & \\
$0-5$ & $19(37.3)$ \\
$6-10$ & $15(29.4)$ \\
$11-15$ & $9(17.7)$ \\
$16-20$ & $4(7.8)$ \\
$20+$ & $4(7.8)$ \\
\hline Have you supervised trainees in the past year? & \\
Yes & \\
No & $38(74.5)$ \\
\hline Number of ultrasound procedures done or supervised in the past year & $13(25.5)$ \\
0 & \\
$1-5$ & $19(37.3)$ \\
$11-20$ & $19(37.3)$ \\
\hline $20+$ & $10(19.6)$ \\
\hline & $2(3.9)$ \\
\hline & $1(2.0)$ \\
\hline
\end{tabular}

In the initial program development, refresher training was encouraged but optional. We intentionally tracked those that completed refresher training compared with those that did not. Based on the results showing significant skills retention among those attending some form of refresher training, the program is planned to change to make this a requirement. We recommend refresher training within 12 months of the initial introductory course. There were several hospitalists that were unable to accommodate taking a full-day refresher course and, therefore, monthly scanning sessions were provided as an alternative.

The main limitation of the study is that it was completed in a single hospital system with available training mentors in $\mathrm{PO}$ CUS. This gave us the ability to perform longitudinal training but may make this less reproducible in other hospital systems. Another limitation is that our course participants did not complete the pre- and postknowledge assessments for the refresher training components of the program, though they did for the initial 3-day course. Our pre- and postassessments have not been externally shown to produce valid data, though they are based on the already validated CHEST ultrasound data. ${ }^{44}$

Finally, our CHAMP Ultrasound Program required a significant time commitment by both faculty and learners. A relatively small percentage of hospitalists have completed the final assessments. The reasons are multifactorial, including program rigor, desire by certain hospitalists to know the basics but not pursue more expertise, and the challenges of developing a skillset that takes dedicated practice over time. We 
have aimed to address these barriers by providing additional hands-on scanning opportunities, giving timely feedback with portfolios, and obtaining more ultrasound machines. We expect more hospitalists to complete the final assessments in the coming year as evidenced by portfolio submissions to the shared online portal and many choosing to attend either the monthly scanning sessions and/or the 1-day course. We recognize that other institutions may need to adapt our program to suit their local environment.

\section{CONCLUSION}

A comprehensive longitudinal ultrasound training program including competency assessments significantly improved ultrasound acquisition skills with hospitalists. Those attending monthly scanning sessions and participating in the portfolio completion as well as a refresher course significantly retained and augmented their skills.

\section{Acknowledgments}

The authors would like to acknowledge Kelly Logue, Jason Robertson, MD, Jerome Siy, MD, Shauna Baer, and Jack Dressen for their support in the development and implementation of the POCUS program in hospital medicine. Disclosure: The authors do not have any relevant financial disclosures to report.

\section{References}

1. Spevack R, Al Shukairi M, Jayaraman D, Dankoff J, Rudski L, Lipes J. Serial lung and IVC ultrasound in the assessment of congestive heart failure. Crit Ultrasound J. 2017:9:7-13.

2. Soni NJ, Franco R, Velez M, et al. Ultrasound in the diagnosis and management of pleural effusions. J Hosp Med. 2015 Dec;10(12):811-816.

3. Boyd JH, Sirounis D, Maizel J, Slama M. Echocardiography as a guide for fluid management. Crit Care. 2016;20(1):274-280.

4. Mantuani D, Frazee BW, Fahimi J, Nagdev A. Point-of-care multi-organ ultrasound improves diagnostic accuracy in adults presenting to the emergency department with acute dyspnea. West J Emerg Med. 2016;17(1):46-53.

5. Glockner E, Christ M, Geier F, et al. Accuracy of Point-of-Care B-Line Lung Ultrasound in Comparison to NT-ProBNP for Screening Acute Heart Failure. Ultrasound Int Open. 2016;2(3):E90-E92.

6. Bhagra A, Tierney DM, Sekiguchi H, Soni NH. Point-of-Care Ultrasonography for Primary Care Physicians and General Internists. Mayo Clin Proc. 2016 Dec;91(12):1811-1827.

7. Crisp JG, Lovato LM, Jang TB. Compression ultrasonography of the lower extremity with portable vascular ultrasonography can accurately detect deep venous thrombosis in the emergency department. Ann Emerg Med. 2010;56(6):601-610.

8. Squire BT, Fox JC, Anderson C. ABSCESS: Applied bedside sonography for convenient. Evaluation of superficial soft tissue infections. Acad Emerg Med. 2005;12(7):601-606.

9. Narasimhan M, Koenig SJ, Mayo PH. A Whole-Body Approach to Point of Care Ultrasound. Chest. 2016;150(4):772-776.

10. Copetti R, Soldati G, Copetti P. Chest sonography: a useful tool to differentiate acute cardiogenic pulmonary edema from acute respiratory distress syndrome. Cardiovasc Ultrasound. 2008;6:16-25.

11. Soni NJ, Arntfield R, Kory P. Point of Care Ultrasound. Philadelphia: Elsevier Saunders; 2015

12. Moore $\mathrm{CL}$, Copel JA. Point-of-Care Ultrasonography. N Engl J Med. 2011;364(8):749-757.

13. Rempell JS, Saldana F, DiSalvo D, et al. Pilot Point-of-Care Ultrasound Curriculum at Harvard Medical School: Early Experience. West J Emerg Med. 2016;17(6):734-740. doi:10.5811/westjem.2016.8.31387.

14. Heiberg J, Hansen LS, Wemmelund $K$, et al. Point-of-Care Clinical UItrasound for Medical Students. Ultrasound Int Open. 2015;1(2):E58-E66. doi:10.1055/s-0035-1565173.

15. Razi R, Estrada JR, Doll J, Spencer KT. Bedside hand-carried ultrasound by internal medicine residents versus traditional clinical assessment for the identification of systolic dysfunction in patients admitted with decompensated heart failure. J Am Soc Echocardiogr. 2011;24(12):1319-1324.

16. Alexander JH, Peterson ED, Chen AY, Harding TM, Adams DB, Kisslo JA Jr. Feasibility of point-of-care echocardiography by internal medicine house staff. Am Heart J. 2004;147(3):476-481.

17. Hellmann DB, Whiting-O'Keefe $Q$, Shapiro EP, Martin LD, Martire C, Ziegelstein RC. The rate at which residents learn to use hand-held echocardiography at the bedside. Am J Med. 2005;118(9):1010-1018.

18. Kimura BJ, Amundson SA, Phan JN, Agan DL, Shaw DJ. Observations during development of an internal medicine residency training program in cardiovascular limited ultrasound examination. J Hosp Med. 2012;7(7):537-542.

19. Akhtar S, Theodoro D, Gaspari R, et al. Resident training in emergency ultrasound: consensus recommendations from the 2008 Council of Emergency Medicine Residency Directors Conference. Acad Emerg Med. 2009;16(s2):S32-S36

20. Jacoby J, Cesta M, Axelband J, Melanson S, Heller M, Reed J. Can emergency medicine residents detect acute deep venous thrombosis with a limited, two-site ultrasound examination? J Emerg Med. 2007;32(2):197-200.

21. Jang T, Docherty M, Aubin C, Polites G. Resident-performed compression ultrasonography for the detection of proximal deep vein thrombosis: fast and accurate. Acad Emerg Med. 2004;11(3):319-322.

22. Mandavia D, Aragona J, Chan L, et al. Ultrasound training for emergency physicians_a prospective study. Acad Emerg Med. 2000;7(9):1008-1014.

23. Koenig SJ, Narasimhan M, Mayo PH. Thoracic ultrasonography for the pulmonary specialist. Chest. 2011;140(5):1332-1341. doi: 10.1378/chest.11-0348.

24. Greenstein YY, Littauer R, Narasimhan M, Mayo PH, Koenig SJ. Effectiveness of a Critical Care Ultrasonography Course. Chest. 2017;151(1):34-40. doi:10.1016/j.chest.2016.08.1465

25. Martin LD, Howell EE, Ziegelstein RC, Martire C, Shapiro EP, Hellmann DB. Hospitalist performance of cardiac hand-carried ultrasound after focused training. Am J Med. 2007;120(11):1000-1004

26. Martin LD, Howell EE, Ziegelstein RC, et al. Hand-carried ultrasound performed by hospitalists: does it improve the cardiac physical examination? Am J Med. 2009;122(1):35-41.

27. Lucas BP, Candotti C, Margeta B, et al. Diagnostic accuracy of hospitalist-performed hand-carried ultrasound echocardiography after a brief training program. J Hosp Med. 2009;4(6):340-349.

28. Mathews BK, Zwank M. Hospital Medicine Point of Care Ultrasound Credentialing: An Example Protocol. J Hosp Med. 2017;12(9):767-772.

29. Critical Care Ultrasonography Certificate of Completion Program. American College of Chest Physicians. http://www.chestnet.org/Education/ Advanced-Clinical-Training/Certificate-of-Completion-Program/Critical-Care-Ultrasonography. Accessed March 30, 2017

30. Mayo PH, Beaulieu Y, Doelken P, et al. American College of Chest Physicians/ Société de Réanimation de Langue Française statement on competence in critical care ultrasonography. Chest. 2009:135(4):1050-1060.

31. Donlon TF, Angoff WH. The scholastic aptitude test. The College Board Admissions Testing Program; 1971:15-47.

32. Ericsson KA, Lehmann AC. Expert and exceptional performance: Evidence of maximal adaptation to task constraints. Annu Rev Psychol. 1996;47:273-305.

33. Ericcson KA, Krampe RT, Tesch-Romer C. The role of deliberate practice in the acquisition of expert performance. Psychol Rev. 1993;100(3):363-406.

34. Arntfield RT. The utility of remote supervision with feedback as a method to deliver high-volume critical care ultrasound training. J Crit Care 2015;30(2):441.e1-e6.

35. Ma OJ, Gaddis G, Norvell JG, Subramanian S. How fast is the focused assessment with sonography for trauma examination learning curve? Emerg Med Australas. 2008;20(1):32-37

36. Gaspari RJ, Dickman E, Blehar D. Learning curve of bedside ultrasound of the gallbladder. J Emerg Med. 2009;37(1):51-66. doi:10.1016/j. jemermed.2007.10.070.

37. Barsuk JH, McGaghie WC, Cohen ER, Balachandran JS, Wane DB. Use of simulation-based mastery learning to improve quality of central venous catheter placement in a medical intensive care unit. J Hosp Med. 2009:4(7):397-403.

38. McGaghie WC, Issenberg SB, Cohen ER, Barsuk JH, Wayne DB. A critical review of simulation-based mastery learning with translational outcomes. Med Educ. 2014:48(4):375-385.

39. Guskey TR. The essential elements of mastery learning. J Classroom Interac. 1987;22:19-22.

40. Ultrasound Institute. Introduction to Primary Care Ultrasound. University of South Carolina School of Medicine. http://ultrasoundinstitute.med.sc.edu/ Ulcme.asp. Accessed October 24, 2017

41. Society of Critical Care Medicine. Live Critical Care Ultrasound: Adult. http:// 
Mathews et al | The Comprehensive Hospitalist Assessment and Mentorship with Portfolios (CHAMP) Ultrasound Program

www.sccm.org/Education-Center/Ultrasound/Pages/Fundamentals.aspx. Accessed October 24, 2017.

42. Castlefest Ultrasound Event. Castlefest 2018. http://castlefest2018.com/. Accessed October 24, 2017

43. Office of Continuing Medical Education. Point of Care Ultrasound Work- shop. UT Health San Antonio Joe R. \& Teresa Lozano Long School of Medicine. http://cme.uthscsa.edu/ultrasound.asp. Accessed October 24, 2017.

44. Patrawalla P, Eisen LA, Shiloh A, et al. Development and Validation of an Assessment Tool for Competency in Critical Care Ultrasound. J Grad Med Educ. 2015;7(4):567-573 